\title{
Clinical and Laboratory Evaluation of the Fit Accuracy of Metal Frameworks of Removable Partial Denture Fabricated from Two Different Pattern Materials
}

\author{
Mohamed Y Abdelfattah 1,2 \\ ${ }^{1}$ Department of Prosthodontics, Tanta University, Egypt, ${ }^{2}$ Department of Prosthodontics, Taif University, KSA
}

\begin{abstract}
Objective: The purpose behind this conducted study was clinical and laboratory assessments of the fit accuracies of cobaltchromium frameworks for removable partial dentures fabricated by traditional lost wax technique (TT), and the Light Cure Modeling Material Technique (LCMT).

Materials and Methods: Sixteen healthy male Kennedy class I patients were selected from the specialty clinics in the Faculty of Dentistry, Taif University. The patients were divided into two Groups eight patients each; Group I: their metallic framework was constructed from the traditional lost wax patterns technique (TT), Group II: their metallic framework was fabricated by Light Cure Modeling Material Technique (LCMT). Conventional clinical and laboratory steps for construction of metallic partial denture were followed. The frameworks resulted from the two pattern materials were tested clinically and laboratory for fit accuracies and the results were statistically analyzed using the two-tailed t-tests.

Results: Clinical results show an appropriate thickness of the impression materials and improved fit accuracy of the Light cure group in comparison to the traditional wax pattern group. Laboratory findings also showed that the Light cure group has significantly smaller mean gap values than the traditional wax pattern group.

Conclusions: The fit accuracies of cobalt-chromium frameworks of removable partial denture fabricated from LCMT are better than that fabricated from TT.
\end{abstract}

Key Words: Cobalt-chromium frameworks, Traditional lost wax technique, Light cure modeling material technique

\section{Introduction}

Dentistry is at crossroads and faces immense challenges, among which the most reviewed problem faced is related with a misfit of metallic frameworks which are generally caused due to the inappropriate lab works, improper impression registration, or ridge changes following denture delivery [1]. To minimize these problems; framework try in appointment is called out for checking the fit of the metallic framework both intraorally and on the definitive cast $[2,3]$. It is made sure that prior to placement of the metallic framework intraorally all the components of the metallic framework should accurately fit on the master cast [4].

In spite of being well-known pattern material, wax leads to dimensional inaccuracies during casting, due to its soft nature and high ductility [5]. Several pattern materials and techniques have been created for fabrication of PRDP; lightpolymerized patterns is among one of them with the advantages of (i) High strength, (ii) Lower flow than pattern wax, (iii) Excellent elastic recovery, (iv) Non-residue burnout, (v) Prolonged time to construct the pattern, (vi) Improved accuracy, and (vii) Firmness following polymerization [6-8]. Light-polymerized patterns can be constructed directly on the master cast, eliminating duplication and refractory cast steps thus saving time and cost. It can also be adjusted after polymerization by rotary instruments and can be evaluated intraorally before being invested [8]. In addition, finite element analysis can also be used to scan, analyze and test the framework design even prior to their fabrication [9].

Improper fit is noted in about $75 \%$ of removable partial dentures and this may lead to teeth movements and discomfort [8]. Poorly fitted dentures can lead to root caries, denture stomatitis, halitosis, oral candidiasis, dental Plaque accumulation, periodontitis and some systemic diseases [10]. Light-polymerized pattern material has wax-like components and encloses acrylates, and fillers [11].

Kumar et al. [12] recognized that the fit of the light-cured clasp assembly was higher than that fabricated by conventional lost wax technique. Takaichi et al. [13] presented a novel method for the construction of metallic framework by the light-polymerizing plastic pattern. They concluded that this material has clinically acceptable accuracy but needed an additional evaluation of the dimensional stability of the prosthesis.

The purpose of this work was clinical and laboratory assessments of the fit accuracies of cobalt-chromium frameworks for removable partial dentures fabricated by traditional lost wax technique (TT), and the Light Cure Modeling Material Technique (LCMT).

\section{Material and Methods}

Sixteen healthy male Kennedy class I patients aged between 45-65 years were selected from the specialty clinics for the study by the Faculty of Dentistry, Taif University. These selected patients were then grouped into two groups of 8 patients each. Patients who belonged to Group I their metallic framework were fabricated by traditional lost wax pattern technique (TT) (BEGO, Germany), and Patients belonging to Group II their metallic framework were fabricated by Light Cure Modeling Material Technique (LCMT) (Metacon light cured dental wax system; primotec USA) and all the steps were constructed by the same dental laboratory technician. Conventional clinical and laboratory steps for construction of metallic partial denture were followed. For each patient, primary alginate impressions (Ivoclar Vivadent, 
Liechtenstein) were taken and poured to produce the study casts which were surveyed and used to construct special tray in acrylic resin (Acrostone, Acrostone Dental, and Medical Supplies Egypt). The design was standardized for all frameworks in both groups; consisted of a lingual plate major connector, two RPI clasps, and meshwork minor connectors. The mouth preparations were done and secondary impressions (Silicon Rubber Base Impression Material, Coltene, Switzerland) were taken and poured in extra hard stone (Bego Stone Plus, BEGO, Germany)

\section{Laboratory steps to construct frameworks by the conventional lost wax technique}

The master casts were surveyed and duplicated by using agaragar with a low melting point $\left(80^{\circ}\right)$ (Ruthinium Stargel; Dental Manufacturing SPA, Italy) to produce precise refractory casts. Phosphate-bonded investment material (Wirovest; BEGO USA Inc.) was mixed, spatulated under vacuum, and then poured into the agar mold and left for one hour to set. Then the casts were separated from the duplicating material and left 45 minutes to dry in an oven at $93^{\circ} \mathrm{C}$. The traditional laboratory procedures to produce metal framework were standardized in all frameworks; the refractory casts were dipped for 15 to 20 seconds in $149^{\circ} \mathrm{C}$ clean high-quality beeswax (Golden beeswax granules, Huading, Henan, China); after that, the casts were left to cool and ready-made wax pattern materials (BEGO; Germany), were adapted on the refractory cast (Figure 1); spruing; investing with phosphatebonded investment material (Silikan $^{\mathrm{TM}}$, SpofaDental, Kerr, Czech Republic); wax burnout; Casting cobalt-chromium alloy (Dedeco, Success Dental Company, Hong Kong ) in a casting machine (Fornax ${ }^{\circledR}$ T-BEGO USA Inc.) $1200^{\circ} \mathrm{C}$. Then the frameworks were devested and cleaned by using airborneparticle abrasion with aluminum oxide $(50 \mathrm{~mm})$ for 15 seconds and fitted on the stone master cast.

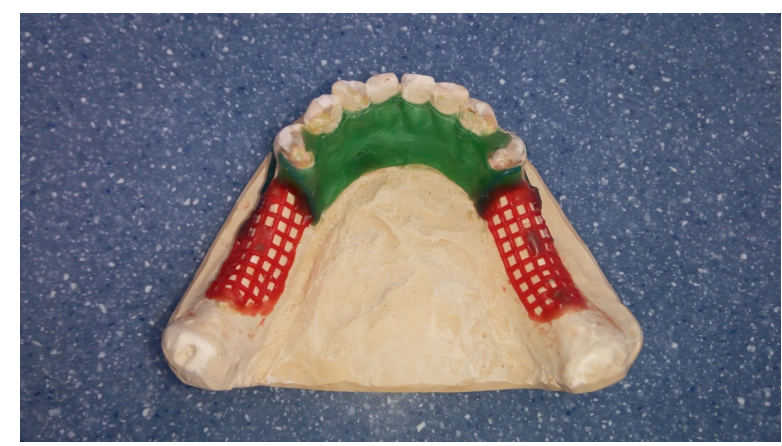

Figure 1. Traditional lost wax pattern technique.

\section{Laboratory steps to construct frameworks by light cure modeling pattern material technique (LCMT)}

All the steps were standardized in all frameworks. Metacon Step I (primotec USA) was applied for 50 seconds to seal the surface of the stone cast then Metacon Step II (primotec USA) was applied for 30 seconds for complete isolation of the master cast. Readymade Metacon light cured dental wax system (primotec; USA) was directly adapted on the stone master cast. $0.9 \mathrm{~mm}$ wrought wires were used to support the plastic frameworks. Then the plastic frameworks were cured for 6 minutes in a light curing unit (Figure 2). The pattern surfaces were conditioned by A Metacon finish varnish and then light-curing was repeated for $2 \mathrm{~min}$. After that, the plastic frameworks were sprued, invested, burned out, casted, and fitted similar to the traditional technique [14].

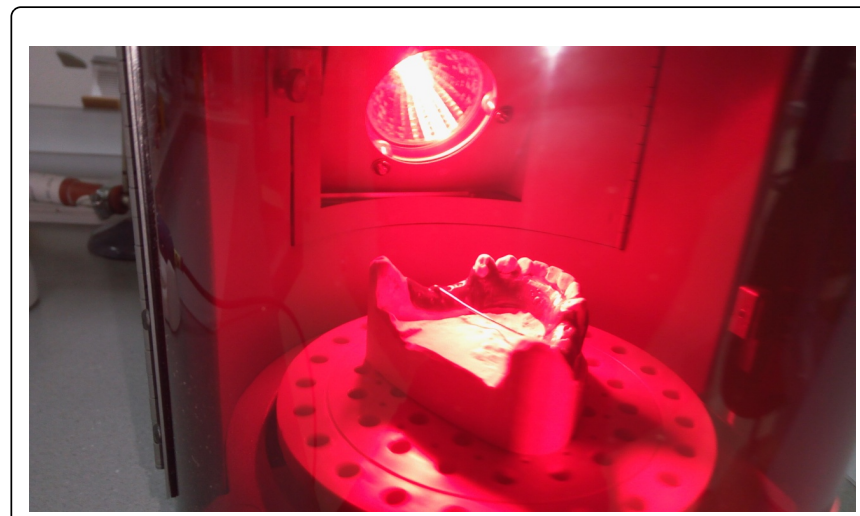

Figure 2. Light cure wax pattern inside the curing unit.

\section{Measurement of Fit Accuracy}

\section{Laboratory evaluation}

The frameworks were then returned back to their stone master casts.

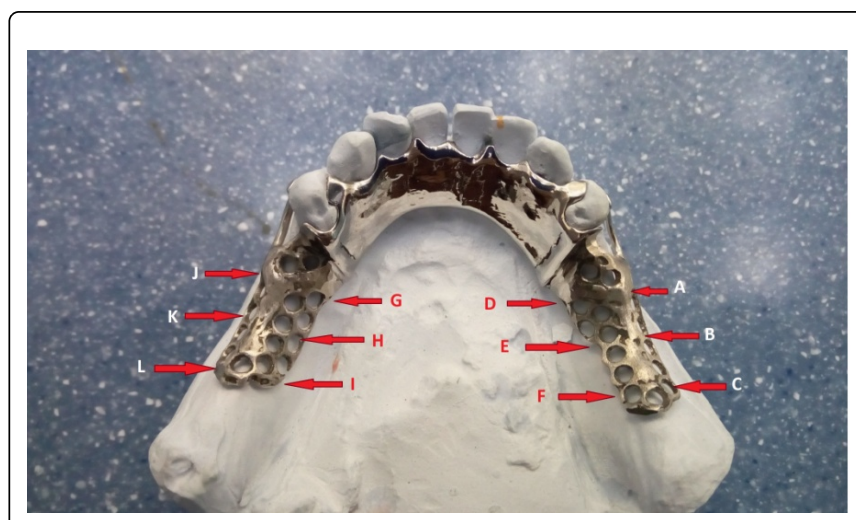

Figure 3. Three gap measurement sites at each side (arrows).

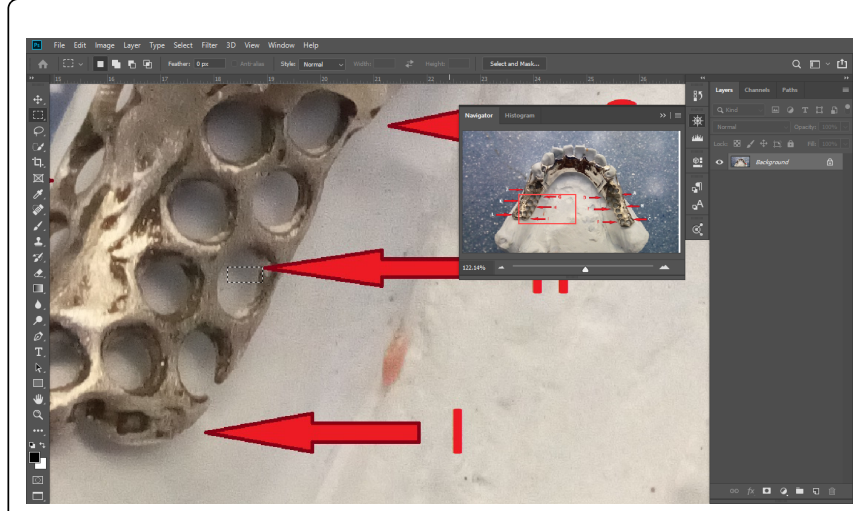

Figure 4. A Photoshop screen image showing the width of the measured gap. 
The gap spaces between the crest of the alveolar ridge of the stone master cast and metal saddle of the framework were measured at three points on each side (Figure 3).

A USB digital 500-megapixel resolution, waterproof Wi-Fi intraoral camera (Henan Dengteer Medical Co., Ltd., China) and a focus Range of $10 \mathrm{~mm}-50 \mathrm{~mm}$ was used to capture images at X16 magnification. All images were captured by the same examiner. To identify the measurement accuracies, a ruler was placed above the stone cast during image capturing. A graphics editing program (Adobe Photoshop CC, 2017 Adobe Systems Inc., San Jose, CA, USA) was used to measure the gap distance at three points per side (Figure 4).

\section{Statistical analysis}

96 gap measurements were done; (48 measurements for TT group and for LCMT group). For each group, the Mean gap measurements and Standard Deviations (SDs) were calculated and SPSS version 20 (SPSS, Chicago, IL) was used for statistical comparison of the traditional technique and Light Cure Modeling pattern Techniques, through the two-tailed ttests at 5\% significance level.

\section{Clinical evaluation}

The edentulous area on the master cast was lubricated and Polyvinyl siloxane (PVS) occlusal registration material (Virtual CADbite, Ivoclar Vivadent AG, Liechtenstein) was applied to it then the framework was placed onto the cast [15] (Figure 5) ensuring complete seating of minor connectors, indirect retainers and rests on their position on the master cast. After setting of the PVS, the frameworks with the registration material were removed and excess materials were trimmed and a light body rubber base impression material was injected on the fitting surface of the PVS material and immediately placed in the patient mouth ensuring that all the components were in their position intraorally. After setting of the impression material, the frameworks were removed from the mouth and careful measurements of the thickness of the impression materials at different locations were performed by a single examiner [16]. The resulted measurements were statistically analyzed using the two-tailed t-tests.

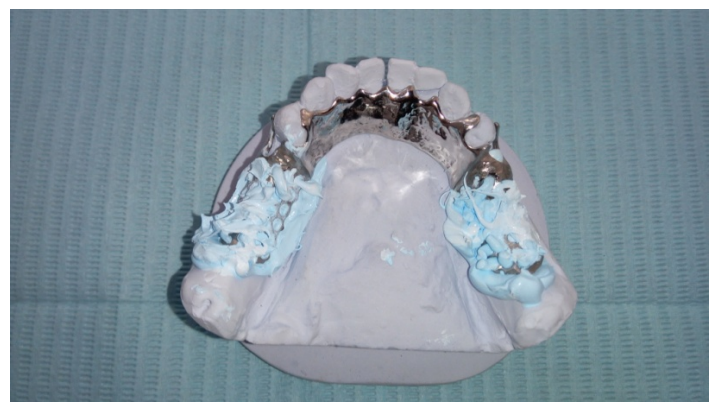

Figure 5. Framework placed over polyvinyl siloxane registration material.

\section{Results}

Inspection of the resulted frameworks exposed that both techniques produced frameworks with excellent quality, without gross surface roughness or casting defects.

\section{Laboratory evaluation}

Table 1 illustrated the Mean measured gaps and standard deviations in (micrometer) of both TT and LCMT groups. There was a statistically significant difference $(p=0.03)$ in the mean gap values of the LCMT and the TT group.

Table 1. Mean measured gaps and standard deviations in (micrometer) of both groups.

\begin{tabular}{|l|l|l|l|l|l|}
\hline Group & $\begin{array}{l}\text { Measured } \\
\text { gaps }\end{array}$ & Mean \pm SD & Maximum & Minimum & P-value \\
\hline Group I & 48 & $\begin{array}{l}160.72 \quad \pm \\
44.6\end{array}$ & 420 & 25 & \\
\hline Group II & 48 & $\begin{array}{l}117.89 \quad \pm \\
39.2\end{array}$ & 311 & 20 & $0.03^{*}$ \\
\hline
\end{tabular}

TT: Traditional lost wax technique

LCMT: Light-curing modeling material technique

\section{Clinical evaluation}

Table 2 showed the Mean impression thickness and standard deviations in $(\mathrm{mm})$ of both groups. The mean thickness values were statistically significant $(\mathrm{p}=0.04)$ in the TT group in relation to the LCMT group.

Table 2. Mean impression thickness and standard deviations in (mm) of both groups.

\begin{tabular}{|l|l|l|}
\hline Group & Mean \pm SD & P-value \\
\hline TT Group & $1.7 \pm 0.06$ & \multirow{2}{*}{$0.04^{*}$} \\
\cline { 1 - 2 } LCMT group & $0.99 \pm 0.04$ & \\
\cline { 1 - 2 } *Statistically significance at $(p>0.05 \%)$ & \\
\hline
\end{tabular}

TT: Traditional lost wax technique

LCMT: Light-curing modeling material technique

\section{Discussion}

Conventional waxes, of course, have different physical and chemical composition than that of the light cure pattern materials.

Several dimensional changes such as syneresis and imbibition's can occur with agar-agar duplicating material thus negatively influence the accuracy of the working cast [12]. light cure material is directly applied on the master cast without duplication, hence, so it is logic that fit accuracy of frameworks fabricated from the LCMT is better than those fabricated from TT pattern materials.

The findings of this study revealed that the fit accuracy of the frameworks fabricated from the LCMT was higher than that fabricated from TT pattern materials. The fit accuracy at 
the saddle area affects the adaptation of the whole framework [8]. Clinical and laboratory measurements of the gap spaces between the crest of the alveolar ridge of the stone master cast and the saddle part of the metal frameworks were evaluated to establish the accuracy of the metal frameworks produced by both TT and LCMT. It is difficult to achieve optimal fit by both techniques because of the casting and solidification shrinkage of cobalt-chromium alloys [17]. In addition to the expansion of the refractory material and faults during wax block out and duplication [18]. Solidification and cooling contraction of the cobalt-chromium alloy may not be compensated by the expansion of the investment material [19]. Some differences in the fit of the framework will take place even with perfect lab works. Modern advances in the materials and techniques have decreased these inaccuracies, but have not eliminated them [8].

The results of this study were in concurrence with those of Kumar et al. [12] who concluded that the fit accuracy of cast clasps fabricated from the light-cured patterns was significantly better than that made from the conventional wax patterns. While these findings were in contrast with those of Rijuta Virmani et al. [19] who found that there was no statistically significant difference between the dimensional accuracy (dimensional fit, linear dimensional change) of the frameworks fabricated using conventional casting wax and light cure pattern materials. The dimensional fit obtained was higher with that of the conventional group, whereas the linear dimensional change was observed lesser with the light cure group; however, the difference was insignificant $[19,20]$.

Additional studies are required to assess the fit accuracy of other framework components to identify the precise site of the interference. In addition, the effect of the length of the edentulous span on the fit accuracy of the metal framework should be evaluated.

\section{Conclusion}

The frameworks constructed by both techniques TT and LCMT had excellent quality, without gross surface roughness or casting defects. Laboratory evaluation represented that there was a statistically significant difference $(p=0.03)$ in the mean gap values of the LCMT and the TT group while clinical evaluation revealed that the mean thickness values were statistically significant $(p=0.04)$ in the TT group in relation to the LCMT group. So, the fit accuracy of the LCMT is better than that of the TT.

\section{References}

1. Arafa KA. Assessment of the fit of removable partial denture fabricated by computer-aided designing/computer aided manufacturing technology. Saudi Medical Journal. 2018; 39: 17-22.

2. Wong MT, Calverley MJ, Nagy WW. Removable partial denture framework try-in. Journal of Prosthetic Dentistry. 1993; 69: 363-368
3. Young L Jr. Try-in of the removable partial denture framework. Journal of Prosthetic Dentistry. 1981; 46: 579-580.

4. Sato Y, Shimodaira O, Kitagawa N. Systematic clinical evaluation and correction procedures for support of removable partial dentures. Journal of Prosthodontics. 2008; 17: 228-232.

5. Viswambaran M, Sundaram RK. Effect of storage time and framework design on the accuracy of maxillary cobalt-chromium cast removable partial dentures. Contemporary Clinical Dentistry. 2015; 6: 471-476.

6. Bortun C, Sandu L, Porojan S. Wax up failures in the removable partial dentures technology using light curing materials. European Cells and Materials. 2006; 11: 17.

7. Sakaguchi RL, Powers J M. Craig's restorative dental materials. St Louis: Mosby. 2012; 13: 199-251.

8. Phoenix RD, Cagna DR, De Freest CF. Stewart's clinical removable partial prosthodontics. Quintessence Publishing Co, China. 2003; 3: 95-337.

9. Bortun C, Cernescu A, Faur N, Sandu L, Topala F. Reverse engineering and finite element analysis in removable partial denture. European Cells and Materials. 2007; 13: 27.

10. Yang Y, Zhang H, Chai Z, Chen J, Zhang S. Multiple logistic regression analysis of risk factors associated with denture plaque and staining in Chinese removable denture wearers over 40 years old in Xi'an-a cross-sectional study. PLoS One. 2014; 9: 87749.

11. Stephen DC, Lyndon C, Helen C, Brian N, Sue HP, et al. Removable partial dentures: The clinical need for innovation. Journal of Prosthetic Dentistry. 2017; 118: 273-280.

12. Kumar MV, Murugesan K, Bhagath SN. The accuracy of fit of cast clasps designed with conventional wax pattern and light cured patterns: a comparative in vitro study. SRM Journal of Research in Dental Sciences. 2010; 1: 10-13.

13. Takaichi A, Wakabayashi N, Igarashi Y. Prefabricated lightpolymerizing plastic pattern for partial denture framework. Contemporary Clinical Dentistry. 2011; 2: 402-404.

14. Anan MT, Al-Saadi Mohannad H . Fit accuracy of metal partial removable dental prosthesis frameworks fabricated by traditional or light curing modeling material technique: An in vitro study. Saudi Dental Journal. 2015; 27: 149-154.

15. Hsu YT. Use of polyvinyl siloxane material for an altered cast impression tray. Journal of Prosthetic Dentistry. 2014; 112: 695-696.

16. Hsu YT. A technique for assessing the fit of a removable partial denture framework on the patient and on the definitive cast. Journal of Prosthetic Dentistry. 2016; 116: 630-631.

17. Anusavice KJ, Shen C, Rawls HR. Phillips' science of dental materials. Elsevier Saunders Inc. 2012; 12: 199-386.

18. Alan BC, David TB. McCracken's removable partial prosthodontics. Elsevier Saunders Inc. 2016; 13: 112-125.

19. Rijuta V, Anandkumar GP, Raghunath P, Prashant AK. Comparative evaluation of the dimensional accuracy of cast partial denture frameworks fabricated by a conventional technique and light-cure wax technique: An in vitro study. Indian Journal of Health Sciences and Biomedical Research KLEU. 2018; 11: 155-159.

20. Swelem AA, Abdelnabi MH, Al-Dharrab AA, Abdel Maguid HF. Surface roughness and internal porosity of partial removable dental prosthesis frameworks fabricated from conventional wax and light-polymerized patterns: a comparative study. Journal of Prosthetic Dentistry. 2014; 111: 335-341. 